\title{
A POESIA CHINESA DEFINIDA POR EZRA POUND: UMA PROCURA PELA ORIGEM
}

Raquel de Sá1

\section{RESUMO}

O presente texto apresenta uma reflexão a respeito de uma possível "zona de contato" entre as teorias de tradução poética definidas por Ernest Fenollosa e seu discípulo Ezra Pound, e a literatura em língua chinesa por eles traduzida. Elaborado por Mary Louise Pratt (1991), estudiosa de literatura espanhola, o conceito de zonas de contato considera a literatura como um espaço social em que diferentes culturas encontram-se, confrontam-se ou mesmo se atracam ("grapple”) a partir de relações de poder, geralmente, muito assimétricas. Ao verificarmos a ausência do reconhecimento deste contato por parte das teorias poéticas dos autores supracitados, sobretudo em relação aos questionamentos levantados por sua crítica, passa a ser admitido, cada vez mais, por diversos estudiosos, inclusive de literatura americana, a importância de dialogar e absorver conhecimentos produzidos por outras áreas, como a sinologia, a filologia, a linguística etc. Quanto a isto, torna-se possível produzir um distanciamento crítico e histórico em relação aos preceitos formulados pelo modernismo literário americano, junto a uma reflexão mais ampla sobre o fenômeno da linguagem. A partir deste cenário, também pretende-se esboçar uma metodologia capaz de detectar formulações e referências teóricas, de tradução e linguagem, em Pound, que, quando omitidas, asseguram uma noção de equivalência entre o texto traduzido e o original chinês, e que igualmente ofuscam a análise das zonas de contato. PALAVRAS-CHAVE: Ezra Pound; Shi Jing; Língua Chinesa; Tradução; Zonas de contato.

Quando pensamos nos problemas que envolvem a tradução de uma língua para outra, podemos refletir se a superioridade do tradutor está no "produto final", isto é, no seu estilo e desenvoltura poética, ou se está na fonte, na sua profunda penetração na mente e na arte do autor de partida que forneceu o material inicial para a tradução (KENNEDY, 1958, p. 24). Entretanto, antes de nos aproximarmos destas questões, também poderíamos sondá-las de outro ponto de vista: o da relação existente entre o "produto

1 Raquel de Sá é graduanda no Departamento de Artes Visuais da Escola de Comunicação e Artes da Universidade de São Paulo. A formulação do projeto de Iniciação Científica foi auxiliada pelos encontros do grupo de estudos "China Antiga: Literatura e Filosofia", orientado pela Prof. ${ }^{a}$ Dr. ${ }^{a}$ Ho Yeh Chia (Departamento de Letras Orientais - FFLCH/USP) e pelo Prof. ${ }^{\circ}$ Dr. ${ }^{o}$ João Vergílio Gallerani Cuter (Departamento de Filosofia - FFLCH/ USP), e pelas disciplinas "Língua Chinesa" I e II, ministradas pela Prof. a Dr. ${ }^{a}$ Ho Yeh Chia, no ano letivo de 2018. E-mail para contato: raqldesa@gmail.com 
Zi Yue: revista de graduação de estudos sinológicos. São Paulo: Portal de Revistas da USP, v. 1, n. 1, 2020.

final" e a fonte. Dito de outro modo, de investigarmos a compreensão que se fez do texto de partida e da intenção que, conjuntamente, mobilizaram a tradução, a fim de, por extensão, identificarmos os métodos implícitos ou explícitos utilizados pelo tradutor, bem como suas implicações para o sentido final.

Quando pensamos na teoria de tradução da poesia chinesa do filósofo americano Ernest Fenollosa (1853 - 1908), e na produção poética e tradutológica de língua chinesa de seu discípulo e conterrâneo Ezra Pound (1885 - 1972), talvez a última perspectiva possa nos trazer novas compreensões quanto ao curioso fenômeno que separa a contribuição de ambos, primeiro, no campo da literatura e arte ocidentais, e, segundo, no campo da sinologia. Apesar dos dois haverem sido direta e indiretamente ${ }^{2}$ questionados pelo último sobre a consistência de seus argumentos, em especial devido sua aproximação efetiva com a língua e poesia chinesas, como explicar a falta de absorção dos conhecimentos do último pelo primeiro? Ou mesmo sua resistência? Sobre isso, surgem de tempos em tempos análises que voltam a questionar esta distância, tanto para sustentá-la ${ }^{3}$, quanto para questioná-la ${ }^{4}$, sob o justo pretexto de ser exatamente o objeto

2 A primeira edição do livro Chinese Characters: their origin, etymology, history, classification and signification. A through study from Chinese Documents, traduzida para o inglês por L. Davrout S.J., do jesuíta francês L. Wieger S.J., apareceu pela primeira vez em 1915. Anterior às principais publicações de Pound, e até hoje fonte para estudiosos, a obra traz conceitos formulados por filólogos chineses de categorias de significação dos caracteres chineses. Uma das categorias citadas, baseada em seu uso, é denominada “false borrowing” (假借, jiăjiè ), isto é, em que a pictografia do caractere não se relaciona com o sentido atribuído, por tratar-se ora de uma associação arbitrária, feita por convenção para designar um objeto que tem um nome na língua falada mas nenhum caractere específico, ou por se tratar de um erro de copistas que não fora corrigido com o passar do tempo, tornando-se oficial. "Not a few characters were thus given artificial meanings, besides their own meaning and their different meanings [...]. Other characters, either names of lost things, or useless doubles, first disappeared and then appeared again with a meaning quite new and in absolute contrast with their composition" (WIEGER, 1965, p. 11). 3 Segundo artigo recente, o argumento que justifica a teoria poética e de tradução de Fenollosa, desenvolvida no seu hoje famoso The Chinese Written Character as a Medium for Poetry, escrito no início do século XX, contra as críticas pronunciadas por seus "detratores" (identificados como os "eruditos sinólogos"), sugere que esta só pode ser entendida sob a ótica da "linguagem poética" (DANTAS, 2013, p. 47), pois: "Por fim, não podemos esquecer que o próprio destino do texto manuscrito original nos diz muito sobre como ele deve ser lido. Basta sabermos que, de posse do material deixado pelo marido Ernest, a viúva Mary Fenollosa preferiu confiá-lo não a eruditos ou sinólogos, mas a um jovem poeta, hoje mundialmente conhecido como Ezra Pound." (DANTAS, AMORIM, 2013, p. 48) Mobiliza-se, neste caso, uma associação direta que equaciona a compreensão de uma teoria de tradução com a face poeta do tradutor - que explica, em Pound, a causa de sua posterior fama como tradutor da poesia clássica chinesa. Segundo outro autor, Giorgio Sica, referindo-se a recepção do livro Cathay (1915) - "traduções chinesas, em boa parte atribuídas a Li Po, [...] efetuadas com base nas anotações, às vezes obscuras, às vezes imprecisas, de Fenollosa" (SICA, 2017, p. 150) e publicadas postumamente por Pound -, este entendia que "Embora sua leitura "criativa" dos ideogramas tenha provocado o repúdio dos sinólogos, Pound nos legou, com toda essa precariedade, aquela que George Steiner definiu sua "obra mais inspirada [...] e o trabalho que chega mais perto da justificação de todo programa imagista", contribuindo de maneira fundamental para a renovação do verso inglês" (SICA, 2017, p. 151). E ainda: "Se os sinólogos, por um compreensível espírito de casta, não apreciaram Cathay, a recepção do mundo poético foi calorosa" (SICA, 2017, p. 151). 4 A fim de verificarmos os argumentos de um dos "detratores" mencionados no artigo de Dantas (2013, p. 47), o texto de Yu-Kuang Chu, reconhecido pelo seu trabalho no campo de estudos interculturais, ao tratar das inter-relações entre linguagem e pensamento em chinês, nos apresenta sucintamente algumas características da gramática chinesa, para explicar, como objetivo principal do artigo, conceitos da linguística determinista, "de que a estrutura da linguagem influenciou os processos mentais, embora se pudesse defender a tese contrária", bem como "a maneira que as concepções ocidentais que conseguiram se impor na China moderna levaram às diversas reformas que estão atualmente ocorrendo na língua” (CHU, 1977, p. 234). Assim como Fenollosa identifica que a ausência do verbo "ser" na sentença chinesa implica uma maneira de compreender a natureza e, por conseguinte, de estar mais aproximada dela - "We do not say a tree "greens itself," but "the tree is green"; not that "monkeys bring forth live young," but that "the monkey is a mammal." (FENOLLOSA, 1920, p. 369) -, sugerindo que esta aproximação se dê através do aspecto pictográfico do ideograma, Yu-Kuang Chu, por sua vez, ao explicar a recepção arbitrária dos caracteres 
da obra de Fenollosa e Pound a língua, a filosofia, a poesia e a arte chinesas. Logo, o que permite que estas contribuições permaneçam tão distantes, sem chance de frontalmente se encontrar, contradizer, combater, e após produzir novos desdobramentos em seus respectivos campos do conhecimento? Do lado da literatura e da arte, para uma compreensão mais plausível da língua e da cultura da qual se apropria, de forma a revisitar criticamente os critérios que legitimaram os procedimentos de tradução da poesia ocidental, e, do lado da sinologia, a resposta do pra quê e o porquê fora a língua chinesa a escolhida para figurar na renovação da literatura americana do início do século XX, de maneira que explique uma apropriação por vezes considerada redutora e/ou equivocada, e que perdura até nossos dias5; como nos diz Kennedy: "If this was a new principle in Fenollosa's time, he must be given credit for its forceful presentation, but it is hard to see why it was necessary to shore it up with questionable Chinese props" (KENNEDY, 1958, p. 27).

Assim, diferente do que nos diz George A. Kennedy sobre The Great Digest (1928) de Pound quando "Undoubtly this is fine poetry. Undoubtedly this is bad translation. Pound has the practice, but not the learning. He is to be saluted as a poet, but not as a translator" (1958, p. 36), ou mesmo distante de defendermos arbitrariamente uma correspondência necessária à prática de tradutor junto à de poeta, compreendemos o caso como um problema de alteridade, sempre complexo, uma vez que traduzir necessariamente implica defrontar-se para depois transformar uma diferença: linguística, cultural, social, política, histórica, geográfica, tudo ao mesmo tempo. Diferente de como costumamos conceber a tradução, muitas vezes de forma utilitarista e transmissora de uma informação objetiva, podemos, ao contrário, identificar uma série de escolhas realizadas pelo tradutor que interpretam a diferença: a partir da escolha de palavras isoladamente, modifica-se também o conjunto linguístico maior que ela participa, que, na esteira histórica, foram aderindo acréscimos de conteúdo — e, portanto, contribuições de sentido — , através da

chineses (que na sua totalidade, possui menos de $4 \%$ de origem pictográfica, segundo o dicionário etimológico compilado em 100 d.C.) pelo leitor de língua chinesa, nos apresenta, além de outras variáveis advindas da linguística moderna para entender a relação entre linguagem e realidade, e da inconsistência prática da qualidade pictórica identificada por Fenollosa, o autor não deixa de mencionar a dimensão artística e visual da escrita chinesa: "Essa qualidade pictórica dos caracteres chineses levou Fenollosa (que escrevia no alvorecer do século) a afirmar que ela muito contribuiu para a imagética visual da poesia chinesa. Admitia-se que, ao ver o símbolo da "lua", o leitor chinês não somente obtinha uma ideia da linha, mas como também via uma lua crescente. Tal concepção está hoje desacreditada, simplesmente por não ser verdadeira. Quase todos os caracteres pictográficos modificaram tão drasticamente suas formas que já não são imagens picturais. $\mathrm{O}$ leitor chinês simplesmente os considera como símbolos convencionalizados de ideias. É ainda certo, entretanto, que os chineses tratam os caracteres escritos como desenhos artísticos. Talvez não seja coincidência que a arte chinesa sobressaia no campo visual” (CHU, 1977, p. 240). $5 \quad$ Segundo Michelle Yeh tem argumentado, "Although it is well known that Pound's translation is a particularly free, often ingenious rendition of the Chinese... what neither he nor Eliot could have foreseen was how powerful and lasting this translation would be in shaping poets' and translators' perceptions of Chinese poetry" (YEH, 1994, p. 139 apud WILLIAMS, 2006-2007, p. 23). Cf. Yeh, Michelle. Anthology of Modern Chinese Poetry. New Haven: Yale UP, 1994. Robert Kern similarmente argumenta: "to the extent that any translation deviates from [Pound's] conventions, it runs the risk of violating our sense of what Chinese poetry has in fact come to be" (KERN, 1996, p. 181 apud WILLIAMS, 2006-2007, p. 23) Cf. Kern, Robert. Orientalism, Modernism and the American Poem. Nova Iorque: Press Syndicate of the U of Cambridge, 1996. 
Zi Yue: revista de graduação de estudos sinológicos. São Paulo: Portal de Revistas da USP, v. 1, n. 1, 2020.

inter-relação ininterrupta entre linguagem e realidade, ou, se preferirmos, sociedade. Desta forma, tendo de lidar com a linguagem — e sendo a linguagem com seu conteúdo uma unidade, como uma fruta e sua pele, (BENJAMIN, 1923, p. 79) — , a tradução concomitantemente lida com a construção histórica de seus sentidos. Como nos diz Walter Benjamin, em seu famoso The Task of the Translator de 1923:

For just as the tenor and the significance of the great works of literature undergo a complete transformation over the centuries, the mother tongue of the translator is transformed as well. While a poet's word endure in his own language, even the greatest translation is destined to become part of the growth of its own language and eventually be absorbed by its renewal. Translation is far removed from being the sterile equation of two dead languages that of all literary forms it is the one charged with the special mission of watching over the maturing process of the original language and the birth pangs of its own $(1923, \mathrm{p}$. 78).

Não sendo o escopo deste texto desenvolver a teoria benjaminiana da tradução (e algumas das impossibilidades prescritas pelo autor), aproveitamos sua visão quanto ao aspecto histórico da língua, que a tradução é capaz de "zelar"' quando se propõe a analisar uma língua e seu processo de amadurecimento no que diz respeito, especialmente, ao seu conteúdo.

Desta maneira, além de considerarmos o discurso que aclama a obra de Fenollosa e de Pound, julgamos importante, para nos aproximarmos destas questões, compreender a obra de tradução de Pound quando comparada à sua origem: o chinês clássico e seu conteúdo histórico (métrica, rima, gramática, sintaxe, sonoridade etc.). A fim de reestabelecermos a ordem de ocorrência destas manifestações ${ }^{7}$ e, assim, neutralizar noções de equivalência e transparência ${ }^{8}$ que poderiam existir quando experienciamos uma tradução, permitindo que delineemos as implicações de sentido do método de tradução de Pound. Com uma análise comparativa a outros métodos de tradução da mesma obra de poesia clássica chinesa, realizada por outros tradutores ocidentais influentes (e as respectivas áreas do conhecimento a que se

6 Tradução nossa de parte da citação de Benjamin supramencionada.

7 Para Walter Benjamin, o significado da tradução só pode ser exprimido completamente quando em conexão ao seu original, sendo esta uma das características exclusivas à tradução: "It is plausible that no translation, however good it may be, can have any significance as regards the original. We may call this connection a natural one, or, more specifically, a vital connection. Just as the manifestations of life are intimately connected with the phenomenon of life without being of importance to it, a translation issues from the original - not much from its life as from its afterlife. For a translation comes later than the original, and since the important works of literature never find their chosen translator at the time of their origin, their translation marks their stage of continued life" (1923, p. 76). 8 Na história da teoria de tradução, poderíamos pensar no conceito, definido por Lawrence Venuti, como "autonomy of translation", isto é, "the textual features and operations or strategies that distinguish it from the foreign text and from texts initially written in the translating language. These complicated features and strategies are what prevent translating from being unmediated or transparent communication; they both enable and set up obstacles to cross-cultural understanding by working over the foreign text" (VENUTI, 2002, p. 5). Na medida em que as relações da autonomia da tradução com o texto original mudam, também podemos pensar na categoria de "equivalência": "Equivalence has been understood as "accuracy," "adequacy," "correctness," "correspondece," "fidelity," or "identity"; it is a variable notion of how the translation is connected to the foreign text" (VENUTI, 2002, p.5). A importância de compreender estas categorias, segundo o autor, é que "Yet without some sense of distinctive features and strategies, translation never emerges as an object of study in its own right" (VENUTI, 2002, p.5). 
dedicaram), procuraremos identificar diferentes motivações e métodos de compreensão e adaptação (inevitáveis) realizados na "vida continuada" do texto chinês de partida, que Pound utilizou em seu The Classic Anthology Defined By Conficius (1954), o 诗经 (Shījīng) — poemas compilados durante a dinastia Zhou 週 (zhōu, 1046 a.C. - 256 a.C.) e geralmente traduzido como "Livro das Odes". Acreditamos que, desta forma, não poderemos (pelo menos a princípio) detectar o motivo que anima o apoio e a manutenção dos distanciamentos entre as implicações estruturais da linguagem, produzidas por outras áreas do conhecimento, e a teoria poética de Fenollosa-Pound, mas medir esta distância pela comparação entre diferentes criações literárias e seu original: transformando a efetividade prática das primeiras em falar transparentemente pelo segundo em uma preocupação teórica ${ }^{10}$, nos lançando a uma interpretação da relação entre eles, e não mais do "outro" do qual não compreendemos a língua.

Análises deste tipo, isto é, que ultrapassam o repertório referenciado pela obra traduzida, para realizar comparações e relações com contextos mais abrangentes, como, por exemplo, o fenômeno mais amplo da inter-relação entre linguagem e realidade, ou mesmo as interpretações ideológicas contidas na leitura de algo vasto e complexo como a cultura de um povo (e as consequências deste imaginário para os indivíduos identificados por ela), não só são possíveis, como podemos dizer que se trata de uma preocupação pertinente ao próprio estudo literário.

Ao considerarmos a literatura como um fenômeno não exclusivo em si mesmo, mas exposto e produzido através do contato de seus a(u)tores com seu contexto social, econômico, político, linguístico e social, a estudiosa de língua espanhola, Mary Louise Pratt, propõe que consideremos conjuntamente o caminho inverso: da literatura como agente em seu contexto. Inseparáveis um do outro, literatura e contexto (ou se preferirmos, linguagem e conteúdo), torna-se incontornável não considerar como os reflexos de relações de poder assimétricas existentes numa sociedade, podem proporcionalmente ressurgir na influência de manifestações literárias sobre a realidade; neste sentido, é fácil pensar nos autores autorizados a realizar contribuições e transformações efetivas no conteúdo de um sistema linguístico. Entretanto, ao se interessar pelos sentidos elaborados por autores que tiveram seu poder de atuação na sociedade corrompido, e que, portanto, não puderam ser considerados quando das mudanças contextuais em que estão inseridos, Pratt formula o conceito de "contact zones":

I use this term to refer to social spaces where cultures meet, clash, and grapple with each other, often in contexts of highly asymmetrical relations of power, such as colonialism, slavery, or their aftermaths as they are lived out in many parts of the world today.

9 Tradução nossa de parte da citação de Benjamin supramencionada na nota 7.

10 Como nos diz Lawrence Venuti, "a translation theory always rests on particular assumptions about language use, even if they are no more than fragmentary hypotheses that remain implict or anacknowledged" (VENUTI, 2004, p.6) 
Zi Yue: revista de graduação de estudos sinológicos. São Paulo: Portal de Revistas da USP, v. 1, n. 1, 2020.

Eventually I will use the term to reconsider the models of community that many of us rely on in teaching and theorizing and that are under challenge today (1991, p.34).

Através das "zonas de contato", Pratt formula um conceito capaz de não só proporcionar circunscritamente uma equidade social na contribuição do sistema linguístico, e, por conseguinte, uma intervenção momentânea e mais equilibrada na realidade, mas, sobretudo através da análise literária, propõe uma alternativa à reprodução e à manutenção de um discurso convencionado — segundo a autora, "atualmente posto em causa" exclusivo da realidade social, para compreendê-lo como apenas um dos muitos pontos de vista possíveis numa zona (espacial e virtual) de contato. Desta forma, as diferentes leituras de uma obra literária relacionam-se com as posições igualmente diferentes dos leitores nesta zona (PRATT, 1991, p. 37). Ao relativizar a efetividade prática outorgada pelo discurso social dominante, através da identificação de seus princípios ideológicos e teóricos, Pratt consegue tornar seus reflexos na sociedade identificáveis, e também as circunstâncias de desigualdade que permitiram que certas contribuições de conteúdo fossem por ela abafadas. Em relação ao estudo de caso realizado pela autora no ensaio "Arts of the Contact Zone", Pratt analisa um manuscrito peruano, datado da cidade de Cuzco do ano de 1613 (quase quarenta anos após a queda do império Inca pelos espanhóis), assinado por um nome inconfundivelmente andino e indígena: Felipe Guaman Poma de Ayala. Escrito numa mistura de quechua e um "expressivo espanhol agramatical”, o manuscrito endereçava-se ao rei Felipe III da Espanha. O que chama a atenção da autora é que a carta é gigante, com 1.200 páginas: quase 800 páginas de texto escrito e 400 com ilustrações (desenhos com linhas) com legenda, intitulada: A Primeira Nova Crônica e Bom Governo ${ }^{12}$ (PRATT, 1991, p. 33-4).

Refletindo sobre os reflexos contextuais do escrito, Pratt compreende que, de certa forma, o manuscrito funcionou, já que um texto extraordinário fora escrito, a considerar o esforço de um autor andino que vivia num império que não possuía sistema de escrita. Por outro lado, não foi bem sucedido, já que a carta nunca chegou a seu endereço. Sendo isto também parte de sua história literária, e, como nos aponta a autora, consequência de dinâmicas assimétricas numa zona de contato, a contribuição de Guaman Poma nos chega hoje não só como um relato de seu contexto, mas, quando visto através de sua perspectiva própria, torna-se capaz de relativizar o ponto de vista do colonizador quando hoje interpretamos sua contribuição linguística:

11 Tradução nossa de parte da citação de Pratt supramencionada.

12 Título traduzido neste artigo para o português da versão em inglês descrita no texto de

Pratt, "The First New Chronicle and Good Government" (PRATT, 1991, p. 34). Supomos que o título original estivesse escrito na mesma mistura de espanhol e quechua mencionada anteriormente, 
Guaman Poma's New Chronicle ends with a revisionist account of the Spanish conquest, which, he argues, should have been a peaceful encounter of equals with the potential for benifiting both, but for the mindless greed of the Spanish. He parodies Spanish history. Following contact with the Incas, he writes, "In all Castille, there was a great commotion. All day and at night in their dreams the Spaniards were saying 'Yndias, yndias, oro, plata, oro, plata del Piru" ("Indies, Indies, gold, silver, gold, silver from Peru”) (p. 372 do manuscrito apud PRATT, 1991, p. 35).

De forma semelhante, poderíamos considerar contribuições linguísticas diferentes da de Pound para compreendermos a poesia chinesa, a fim de inserirmos mais pontos de vista neste cenário e propor, assim, uma zona de contato. Em seu artigo Decolonizing Cathay: Teaching the Scandals of Translation Through Angel Island Poetry (2006), John R. Williams aponta a importância de considerarmos a obra poética de imigrantes chineses nos Estados Unidos - anteriores, contemporâneos e conterrâneos a Pound —, com a mesma autoridade para compreendermos a "poesia chinesa" ou a "língua chinesa", através de lentes muito mais próximas e familiarizadas com o objeto de estudo em questão.

Assim, quando partimos apenas da obra de Pound ou Fenollosa, dificilmente concebemos que contemporaneamente a eles haviam imigrantes chineses nos Estados Unidos que, inclusive, produziam poesia em chinês. Somadas às circunstâncias desta produção, também não costumamos traçar a distância entre o discurso elevado de Fenollosa e Pound sobre a cultura chinesa com o primeiro ato de exclusão xenofóbica e racista nos Estados Unidos, em $1882^{13}$, voltado exclusivamente para deter a entrada de chineses no país. E quanto a isto, quando lemos as traduções de Pound, não costumamos visualizar a situação política da China da época, que, após duas derrotas nas Guerras do Ópio, vira suas defesas enfraquecidas contra as cada vez mais violentas investidas colonialistas por parte de países da Europa, em especial Inglaterra e Estados Unidos e, depois, Japão, ocasionando a saída desesperada de várias pessoas em busca de melhores oportunidades de vida pra si e sua família. E, ainda, mal conseguimos relacionar a presença e influência da poesia chinesa nas obras mais importantes de Pound, com uma preocupação com a condição de vida dos indivíduos asiáticos imigrantes nos Estados Unidos, considerando, inclusive, que na juventude do poeta em Hailey, Idaho, houvera a presença de um serviçal chinês empregado por sua família (WILLIAMS, 2006, p. 25).

Ao iniciar uma análise comparativa entre poemas de imigrantes chineses ${ }^{14}$ e a poesia de Pound,

13 "The Exclusion Act of 1882 heralded a change in the nation's immigration pattern. Free and unrestricted immigration was replaced by restrictions and racism. For the first time in American history, members of a specific ethnic group were refused entry and admittance to the naturalization process. Only government officials, merchants, students, teachers, visitors, as well as those claiming U.S. citizenship were admitted" (LAI, H. M.; LIM, G.; YUNG, J., 1991, p. 12). 14 Os poemas foram realizados por ocasião de seu aprisionamento desumano em estações de imigração e registrados nas paredes de uma destas, o porto de Angel Island (São Francisco, Califórnia), entre as décadas de 1910 e 1940, e descobertas por acaso apenas nos anos 1970 por um guarda florestal. Cf., sobretudo o relato proferido 
Williams faz uma importante descoberta: os poetas de Angel Island parafraseavam uma série de referências

a Confúcio, lendas e mitologias chinesas, e inclusive a estrutura dos poemas clássicos originais em chinês, que advinham da mesma tradição daqueles traduzidos por Pound em Cathay. Desta forma, poderíamos dizer que ambos os casos se apropriaram da mesma tradição cultural e suas imagens para produzir suas poesias: em Pound, através da tradução, em alguns casos aproveitava-se da temática de batalha de alguns poemas chineses para falar da experiência da primeira guerra mundial ${ }^{15}$; já os poetas de Angel Island partiam da sua falta de prática em poesia e literatura para gravar, nas paredes dos dormitórios de Angel Island em São Francisco, relatos de racismo, maus-tratos, tortura e sofrimentos lá ocorridos: "looseness in rhythm only means that these particular Chinese-language poems more closely resembles the free-verse forms of Pound's translations than did the actual Chinese poems he was reading" (WILLIAMS, 2006, p. 24).

Ao levar estas semelhanças para a aula de literatura de língua inglesa, Williams pergunta aos seus alunos: por que os tradutores de Angel Island não optaram por uma tradução imagista? O que a tradução poundiana poderia fazer em relação ao conteúdo de poemas que dispunham de tais testemunhos? Diferente de Pound, os tradutores de Angel Island preocuparam-se com a precisão do relato das condições de subsistência dos indivíduos lá presos, não se detiveram em explicar, através de notas de rodapé, metáforas compreensíveis apenas à luz das referências tradicionais chinesas, esclarecendo, por conseguinte, as invenções que surgiram a partir delas, bem como as expressões para designar uma realidade antes não prevista pela língua ${ }^{16}$, além do cruzamento com documentos e relatos sobre a vida das pessoas durante e após o encarceramento.

Assim, concluindo, poderíamos dizer que a contribuição de Pound diz respeito a uma definição de "poesia chinesa", que, prevista pela teoria poética imagista, se apresenta como um locus da interação cultural entre Oriente-Ocidente (WILLIAMS, 2006, p. 26). Entretanto, enquanto não compreendida como uma teoria de tradução, e, portanto, quando não esclarecidas as interpretações ideológicas da linguagem e do "outro" implícitas, corremos o risco de igualmente cercear a extensão de sua obra de seu contexto mais amplo e imediato, ao separarmos as definições do autor de "poesia chinesa" de manifestações

por uma das pesquisadoras do caso, Judy Yung, na aula "Chinese Immigration and Poetry at Angel Island and Ellis Island", em 2014, ministrada na The Murphy Institute: Center for Labor, Community and Policy Studies, a respeito da continuidade da pesquisa e da segunda edição do livro, lançado em 2014, Island: Poetry and History of Chinese Immigrants on Angel Island, disponível online: $<<$ https://aaari.info/15-03-06yung/>>. Acesso em 04 de fevereiro de 2019. 15 Cf. o poema "Song of the Bowmen of Shu" In: POUND, E. Translations. New York: New Directions, 1963. (WILLIAMS, 2006, p. 24). Além disso, sobre uma análise desta utilização de Pound dos poemas chineses, Cf., sobretudo: WILLIAMS, J.R. Modernist Scandals: Ezra Pound's Translations of 'the' Chinese Poem. Orient and Orientalisms in US-American Poetry and Poetics. Ed. Sabine Sielke and Christian Kloeckner. Frankfurt: Peter Lang, 2009. p. 145-65. 16 Cf., as análises dos poemas realizadas por Judy Yung na referência supracitada, disponível online: https://aaari.info/15-03-06yung/ Acesso em 04 de fevereiro de 2019. 
mais amplas da poesia escrita em língua chinesa — justamente para melhor compreendermos o que faz Pound com objeto do qual se apropria, a considerar a influência de sua obra sobre o imaginário ocidental.

Como nos diz Williams ao final de seu texto, sobre a relação entre linguagem e realidade: "To teach the scandals of translation then is to teach the construction of reality" (WILLIAMS, 2006, p. 28). Ao não reproduzirmos as definições de Pound para compreendermos a língua chinesa, também não nos limitamos à realidade que elas nos dão a conhecer. Daí, a poesia que escapa à sua teoria, a tradução poética que também se diferencia dela, e a análise dos sentidos que não são preservados na passagem de uma língua e outra, podem nos apresentar outras realidades. Compará-las, e propor um confronto numa zona de contato, não só relativiza o discurso canônico, expõe seus mecanismos de significação, compõe mais faces do fenômeno da língua chinesa, mas também pode nos auxiliar no importante esforço de esboçar novos possíveis desdobramentos em campos do conhecimento antes distanciados.

\section{REFERÊNCIAS BIBLIOGRÁFICAS}

LAI, H. M.; LIM, G.; YUNG, J. Poetry and History of Chinese Immigrants on Angel Island, 1910-1940. Reprint. Originally Published: Sao Francisco: HOC DOI, 1980. University of Washington edition first published in 1991. BENJAMIN, W. The task of the translator: an introduction to the translation of Baudelaire's Tableaux Parisiens. 1923. Tradução: Harry Zohn. In: The Translation Studies Reader. Second Edition. Organizador: Lawrence Venuti. Routledge: 2002. p.75 - 85. CHU, K.Y. A interação entre linguagem e pensamento em chinês. In: Ideograma: lógica, poesia, linguagem. Tradução Heloysa de Lima Dantas. Organizador: Haroldo de Campos. São Paulo: Cultrix, Ed. Da Universidade de São Paulo, 1977. DANTAS, M. L.; AMORIM, F.F. Fenollosa: da exegese do ideograma às vanguardas. Revista Texto poético. V. 14, p. 39-49, $1^{\circ}$ semestre 2013.

FENOLLOSA, E. An essay on THE CHINESE WRITTEN CHARACTER by late ERNEST FENOLLOSA, edited by Ezra Pound. In:POUND, E.;FENOLLOSA, E. Instigations of Ezra Pound,together with an essay on the Chinese written character by Ernest Fenollosa. Nova Iorque: Boni and Liveright, 1920.p. 357-388. PRITT, M. L. Arts of the Contact Zone. Profession, pp. 33-40, 1991.

SICA. G. O vazio e a beleza - de Van Gogh a Rilke: como o Ocidente encontrou o Japão. Tradução: 
Letizia Zini, Valéria Vicentini. Campinas: Editora Unicamp, 2017.

KENNEDY, G. A. Fenollosa, Pound and the Chinese Character. Yale Literary Magazine, vol. 126, n. 5, p. 24-36, Dezembro 1958.

VENUTI, L. The Translation Studies Reader. Second Edition. Organizador: Lawrence Venuti. Routledge: 2002.

WIEGER, S.J.L. The Chinese characters: their origin, etymology, history, classification and signification. Tradução de S.J. L. Davrout. 2 ed. Nova Iorque: Dover Publications, 1965.

WILLIAMS, J.R. Decolonizing Cathay: Teaching the Scandals of Translation through Angel Island Poetry. Transformations. V. XVII, n.2, p. 15-30, Outono 2006- Inverno 2007. 